\title{
Fabrication of thin films for a small alternating gradient field magnetometer for biomedical magnetic sensing applications
}

\author{
N. J. Jones, ${ }^{1, a)}$ K. L. McNerny, ${ }^{1}$ V. Sokalski, ${ }^{1}$ M. Diaz-Michelena, ${ }^{2}$ D. E. Laughlin, ${ }^{1}$ \\ and M. E. McHenry ${ }^{1,3}$ \\ ${ }^{1}$ Department of Materials Science and Engineering, Carnegie Mellon University, Pittsburgh, \\ Pennsylvania 15213, USA \\ ${ }^{2}$ Payloads and Instrumentation Area, INTA-Instituto Nacional de Técnica Aeroespacial, \\ Ctra. de Torrejón a Ajalvir km 4.2, Madrid, Spain \\ ${ }^{3}$ Department of Biomedical Engineering, Carnegie Mellon University, Pittsburgh, Pennsylvania 15213, USA
}

(Presented 18 November 2010; received 25 September 2010; accepted 2 November 2010; published online 23 March 2011)

\begin{abstract}
Thin film alternating gradient field magnetometers (AGFM) have potential for measuring magnetic moments of minerals in extraterrestrial soil samples. AGFM sensors offer increased spatial resolution required to detect magnetic nanoparticles for biosensing applications. We have fabricated a patterned thin film with the properties necessary for use in a small AGFM system. Hexagonalclose-packed CoCrPt thin films of 20 and $500 \mathrm{~nm}$ were sputtered (nominal composition of $\mathrm{Co}_{66} \mathrm{Cr}_{15} \mathrm{Pt}_{19}$ ), showing a high magnetic moment and large out-of-plane anisotropy. The films showed a $\Delta \theta_{50}$ of better than $3^{\circ}$ for the (002) CoCrPt peak for all films, which improves with thickness. The texture is partly due to the NiW and Ru underlayers. The films showed an out-of-plane easy axis, indicating a strong uniaxial anisotropy that exceeds the shape demagnetization energy. This is due to the addition of $\mathrm{Cr}$, which decreases the magnetic moment of the films; magnetoelastic coupling and film stresses may also aid in achieving a perpendicular anisotropy. The first-order uniaxial anisotropy constants were calculated as a function of temperature, ranging from $3.7 \times 10^{6}$ $\mathrm{ergs} / \mathrm{cm}^{3}$ at room temperature to $6.8 \times 10^{5} \mathrm{ergs} / \mathrm{cm}^{3}$ at $500{ }^{\circ} \mathrm{C}$, and the $T$ dependence agrees with Akulov's theory for uniaxial materials. The thickest film was etched with a checkerboard pattern to decrease the demagnetization effects, which are seen more influentially in the thicker films. This opened up the hysteresis loop, and decreased the amount of field necessary to overcome the thin film geometry. @ 2011 American Institute of Physics. [doi:10.1063/1.3536672]
\end{abstract}

\section{INTRODUCTION}

Magnetic nanoparticles are being explored as tissuespecific tags. However, a sensitive means of measuring small magnetic moments must be developed to detect dilute concentrations of nanoparticles in biological systems. ${ }^{1}$ Thin film alternating gradient field magnetometers (AGFM) composed of permanent magnets are being developed for measuring magnetic moments in soil samples in Martian and Lunar missions. $^{2,3}$ These gradiometers can measure small moments with great sensitivity, and thus will be used in the development of an AGFM for biomedical applications.

An AGFM prototype developed by INTA (Instituto Nacional de Técnica Aeroespacial) employs a sensor head of a square Si membrane with a cylindrical SmCo permanent magnet. ${ }^{2,3}$ The magnetic material attached to the membrane needs sufficient volume to oscillate.

Here, Co-Cr-Pt (see Refs. 4 and 5) was chosen as the sensor magnetic material because of its large magnetic moment $(\sim 425 \mathrm{emu} / \mathrm{cc})$, corrosion resistance, and potential for large perpendicular anisotropy. ${ }^{6}$ A checkerboard pattern was etched into the film to decrease the demagnetizing field associated with thin film geometries.

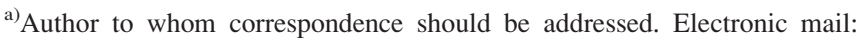
njj@andrew.cmu.edu.
}

\section{PROCEDURE}

Well textured hcp CoCrPt (001) films were deposited on naturally oxidized $\mathrm{Si}$ substrates using a $\mathrm{Ru}(20 \mathrm{~nm}) / \mathrm{Ni}_{90}$ $\mathrm{W}_{10}(10 \mathrm{~nm}) / \mathrm{Ta}(3 \mathrm{~nm})$ underlayer stack (nominal alloy composition of $\left.\mathrm{Co}_{66} \mathrm{Cr}_{15} \mathrm{Pt}_{19}\right)$. Ta serves as an amorphous adhesion layer while the $\mathrm{NiW}$ and $\mathrm{Ru}$ naturally grow with fcc(111) and hcp(001) orientations, respectively, to induce a strong (001) orientation of CoCrPt.

Samples were prepared using a Leybold-Heraeus Z400 RF sputtering system with the base pressure at better than $1 \times 10^{-7}$ Torr. Substrates were cleaned ultrasonically and layers were deposited at room temperature with a working pressure of $5 \mathrm{mT}$ Ar. Deposition rates were determined by step profilometry and ranged from 5 to $8 \mathrm{~nm} / \mathrm{min}$.

A photolithographic technique was used to fabricate the checkerboard pattern with $20 \mu \mathrm{m} \times 20 \mu \mathrm{m}$ feature sizes. A $2.1 \mu \mathrm{m}$ photoresist was spin coated onto the films and prebaked in vacuum at $95^{\circ} \mathrm{C}$ for $2 \mathrm{~min}$. An emulsion transparency mask was placed emulsion side down onto a mask holder GCA 4800 stepper. The exposure was performed using $g$-line UV light, the mask was reduced with a $10 \times$ lens, and subsequently developed. The etch was performed in a Commonwealth Scientific Ion Beam Etching System. The hardened surface layer of photoresist was removed and rinsed in acetone and isopropyl alcohol. 


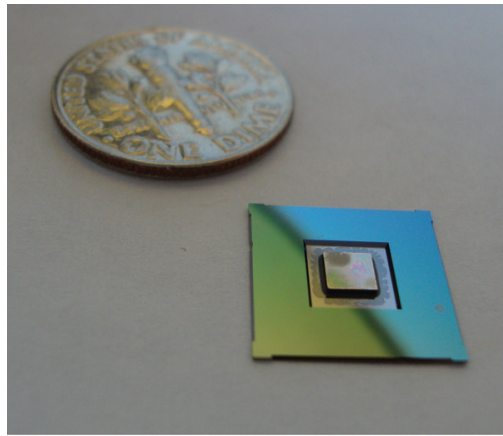

(a)

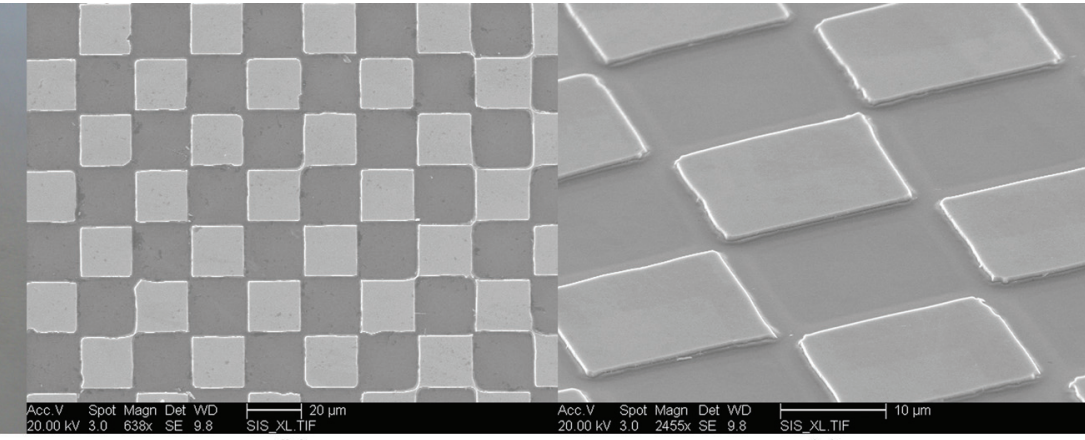

(b)

(c)

FIG. 1. (Color online) (a) Image of a diced, patterned film glued to a Si membrane, and (b-c) SEM images of a checkerboard patterned CoCrPt thin film.

Patterned films were diced into $3 \mathrm{~mm} \times 3 \mathrm{~mm}$ squares (see Fig. 1). Photoresist was spin coated on the surface of the film prior to dicing to protect the device from dicing debris. The squares were rinsed to remove the protective photoresist layer.

Structure, morphology, and magnetic properties were studied using $\mathrm{x}$-ray diffraction (XRD), scanning electron microscopy (SEM), and a physical properties measurement system (PPMS) and vibrating sample magnetometer (VSM).

\section{IIII. RESULTS AND DISCUSSION}

Out-of-plane XRD scans showed good (002) CoCrPt texture. The silicon peak was minimized by a scan offset $(\Omega)$ of $0.1^{\circ}$. To verify the hexagonal crystal structure of the film, the sample was tilted to $\psi=88^{\circ}$ to do an in-plane scan. This scans the planes perpendicular to the film surface, while missing the intense Si peak. The (100) and (200) CoCrPt peaks did not agree with the (002) and (004) peaks from the out-of-plane scan, indicating a break from cubic symmetry and therefore a hexagonal structure.

Using both the in-plane and out-of-plane scans, the lattice parameters were determined to be $a=0.2575 \mathrm{~nm}$, and $c=0.4186 \mathrm{~nm}$, both larger than the bulk Co literature values. $^{7}$ The increase in lattice parameter is due to the dissolution of Pt into the system. Rocking curves in $\Omega$ showed good alignment of the thin film, with $\Delta \theta_{50}$ of the $\mathrm{CoCrPt}(002)$ peak at $2.490^{\circ}$ for the $500 \mathrm{~nm}$ film. The texture alignment was better than that seen in thinner films of the same compo- sition. To understand this rocking curves were also performed on the Ru (002) peak, showing a $\Delta \theta_{50}$ of $3.3^{\circ}$. The hexagonal $\mathrm{CoCrPt}$ films improved with thickness, indicating a preferred (001) hexagonal texture with thickness, which reduces the total surface energy. ${ }^{6}$

The addition of $\mathrm{Cr}$ decreases the alloy saturation magnetization allowing an out-of-plane uniaxial anisotropy. This is seen in Figs. 2(a-c), where the in-plane loops correspond to those of a hard axis. The thinnest film shows the squarest loop. The amount of shearing of the loop is dependent upon the balance between the demagnetization factor and the overall volume of the sample. While both $500 \mathrm{~nm}$ thin films have a slight opening in their hysteresis loops, the hysteresis loop of the checkerboard thin film has opened more and is sheared less than a continuous thin film of the same thickness. This is both due to the decrease in film continuity (exchange coupling) and the decrease in the demagnetization factor, since the edge effects are not as pronounced and the ratio of thickness to side length has been increased.

The opening in the out-of-plane loops in both Figs. 2(b) and 2(c) does not continue through the loop, and this can possibly be attributed to domains, in accordance with Hehn et al. ${ }^{8}$ The difference here, however, would be the preference for out-of-plane anisotropy, which will change the domain structure of the thin film. ${ }^{9}$

The in-plane hard axis loops have a jump near zero field. This is likely due to the alignment of the (002) axis of the hexagonal crystals. While there is very good texture, there

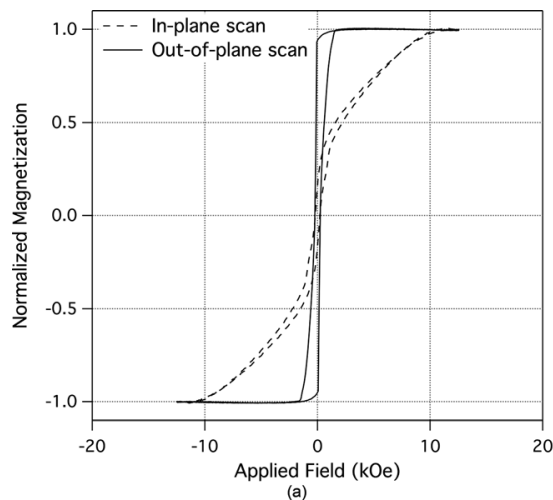

(a)

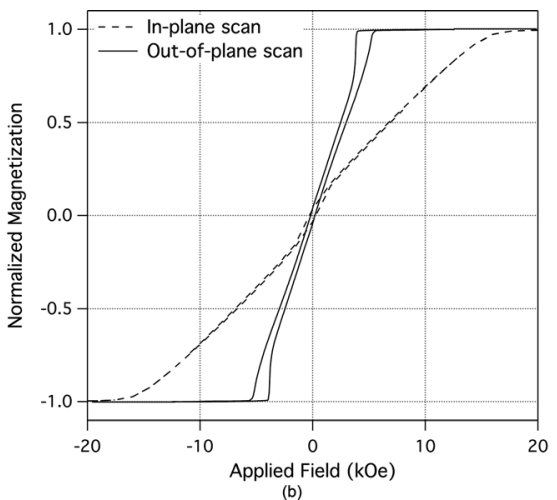

(b)

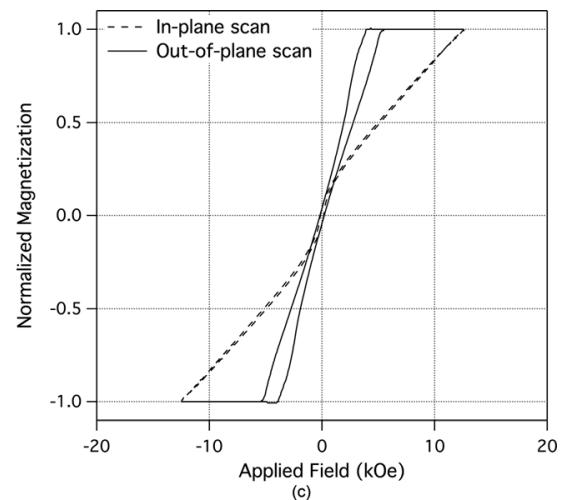

(c)

FIG. 2. Magnetic hysteresis loops with an applied magnetic field oriented parallel and perpendicular to the plane of $15 \%$-Cr CoPt films measured for (a) a continuous $20 \mathrm{~nm}$ sputtered film, (b) a continuous $500 \mathrm{~nm}$ sputtered film, and (c) a checkerboard patterned $500 \mathrm{~nm}$ sputtered film. 
will still be a number of grains not oriented perpendicular to the film surface. These will switch preferentially, causing an initial jump in the magnetization. In agreement with the $\mathrm{XRD}$ rocking curves, the more well-aligned film is the thicker film, which correspondingly has a smaller jump in magnetization near the origin. The in-plane loops show a large anisotropy field of $\sim 1.2 \mathrm{~T}$ for both $500 \mathrm{~nm}$ films.

While there is a second-order anisotropy term that causes curvature in the loops, a simple analysis of these loops can yield a first-order anisotropy term quite easily. There is a difference in curvature between the continuous and checkerboard thin films shown in Figs. 2(b) and 2(c), respectively. This change in curvature can be attributed to a decrease in the exchange coupling of the magnetic moments as they are now separated through the etching process.

The continuous $500 \mathrm{~nm}$ thin films were heated to $1000{ }^{\circ} \mathrm{C}$ in the VSM. Magnetization versus temperature data was collected, along with hysteresis loops at various temperatures.

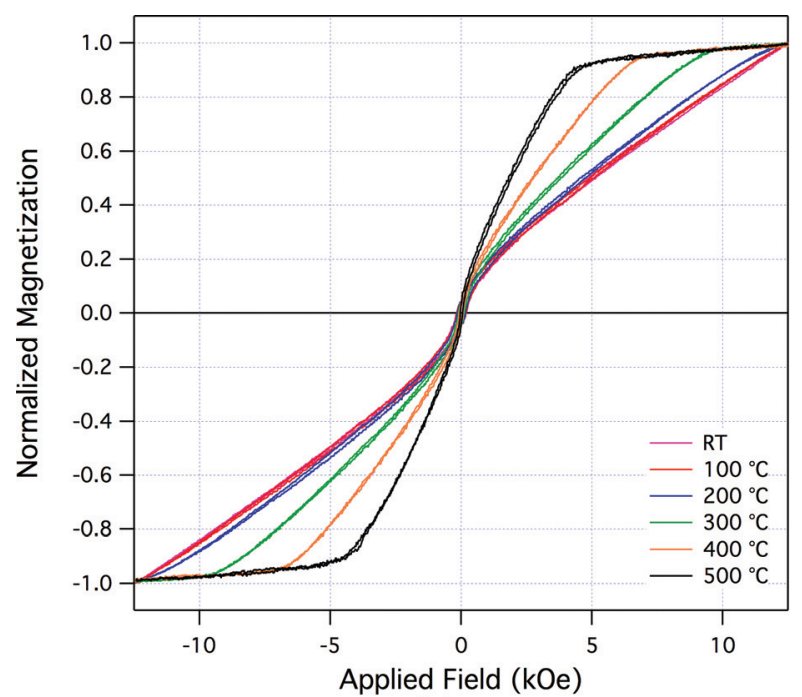

(a)

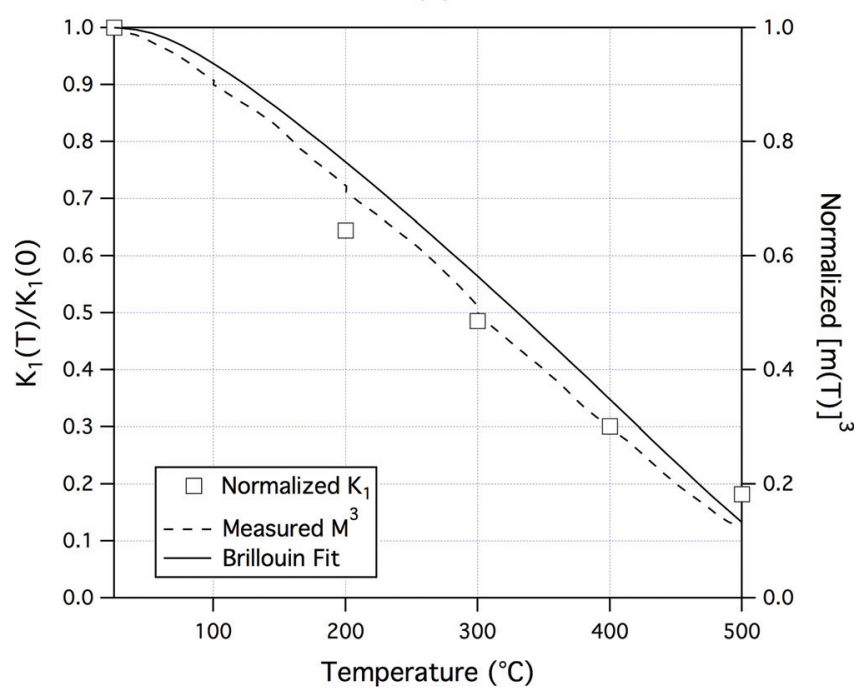

(b)

FIG. 3. (Color online) (a) In-plane hysteresis loops for continuous $500 \mathrm{~nm}$ CoCrPt films measured at temperatures between 25 and $500{ }^{\circ} \mathrm{C}$, and (b) normalized $K_{1}$ values determined from (a) for continuous $500 \mathrm{~nm}$ CoCrPt films plotted as a function of temperature.
Figure 3(a) shows the hysteresis loops up to $500{ }^{\circ} \mathrm{C}$. After $600{ }^{\circ} \mathrm{C}$, the properties of the film began to change and the $M$ vs $T$ curve became discontinuous, indicating oxidation or a change in the morphology of the film. The anisotropy field continuously decreased with increasing temperature. This is due to the $T$ dependence of the effective anisotropy constants, which decreases the strong preference for the (001) magnetization direction. The first-order anisotropy constants can be calculated using the equation $K_{l}=0.5 H_{k} M_{s}$ (assuming no second-order terms), where $K_{1}$ is the first-order anisotropy constant, $H_{k}$ is the anisotropy field, and $M_{s}$ is the saturation magnetization. These values are shown in Fig. 3(b), yielding $3.7 \times 10^{6} \mathrm{ergs} / \mathrm{cm}^{3}$ at room temperature to $6.8 \times 10^{5} \mathrm{ergs} / \mathrm{cm}^{3}$ at $500{ }^{\circ} \mathrm{C}$. These calculations are compared to a plot of the normalized magnetization cubed, and fit reasonably well to the $m(T)^{3}$ dependence of uniaxial anisotropy predicted by Akulov's theory. ${ }^{10,11}$ A Brillouin fit of the $T$ dependence of the magnetization is shown in Fig. 3(b), showing the correct trend and a good agreement with experimental data. If secondorder constants are estimated for room temperature, then $\mathrm{K}_{1}=$ $(2 \pm 0.3) \times 10^{6}$ and $\mathrm{K}_{2}=(3.9 \pm 1.4) \times 10^{5} \mathrm{erg} / \mathrm{cm}^{3}$.

\section{CONCLUSIONS}

CoCrPt thin films have been fabricated with good (001) hexagonal texture. The easy axis is out of plane, with a very square shape in the thinnest film fabricated; the thicker films were sheared due to demagnetization effects and a decrease in internal stresses. Etching the films helped to open the hysteresis loop, and decrease demagnetization effects. Anisotropy constant calculations showed a decreasing trend with increasing temperature that agrees with the theory for uniaxial materials. Given these properties and the good outof-plane texture, the thin films have potential for use in silicon membrane AGFM sensors.

\section{ACKNOWLEDGMENTS}

We thank Carsen Kline for patterning the films, the MSE and BME Capstone groups for their help with sensor assembly, and Marta Duch and José Antonio Plaza of the CNM (Centro Nacional de Microelectrónica) for supplying the silicon membranes. N.J.J. gratefully acknowledges support from a DOD SMART scholarship. N.J.J., K.L.M., M.E.M., and D.E.L. acknowledge support of the NSF through Grant No. DMR \#0804020 and the Data Storage Systems Center.

${ }^{1}$ K. N. Collier et al., J. Appl. Phys. 105, 07A328 (2009).

${ }^{2}$ I. Lucas et al., Sens. Lett. 7, 563 (2009).

${ }^{3}$ I. Lucas et al., Thin-Film Patterned Permanent Magnet Membranes for Micromechanical Susceptometry in Planetary Exploration, 11th Joint MMM/Intermag Conference, Washington, DC. 21 January 2010. Conference Presentation.

${ }^{4}$ L.-L. Lee et al., IEEE Trans. Magn. 31(6), 2728 (1995).

${ }^{5}$ B. Lu et al., J. Appl. Phys. 85(8), 4295 (1999).

${ }^{6}$ M. E. McHenry and D. E. Laughlin, Acta Mater. 48, 223 (2000).

${ }^{7}$ L. J. E. Hofer and W. C. Peebles, J. Am. Chem. Soc. 69, 893 (1947).

${ }^{8}$ M. Hehn et al., Phys. Rev. B 54(5), 3428 (1996).

${ }^{9}$ R. F. Soohoo, Magnetic Thin Films (Harper \& Row, New York, 1965), pp. 30-31.

${ }^{10}$ N. Akulov, Z. Phys. 100, 197 (1936).

${ }^{11}$ B. D. Cullity and C. D. Graham, Introduction to Magnetic Materials, 2nd ed. (IEEE, New York, 2009), pp. 226-229. 